\title{
Computer-Assisted Navigation on the Arrested Heart during CABG Surgery
}

\author{
C. Gnahm ${ }^{1}$, C. Hartung ${ }^{1}$, R. Friedl ${ }^{2}$, M. Hoffmann ${ }^{3}$, K. Dietmayer ${ }^{1}$ \\ ${ }^{1}$ Institute of Measurement, Control and Microtechnology, University of Ulm \\ ${ }^{2}$ Department of Cardiac Surgery, University Hospitals of Ulm and Lübeck \\ ${ }^{3}$ Department of Diagnostic Radiology, University Hospital of Ulm \\ claudia.gnahm@uni-ulm.de
}

\begin{abstract}
Coronary artery bypass grafting (CABG) is the standard treatment for advanced coronary artery diseases (CAD). Optimal placement of the bypass graft on the diseased vessel is very important. To assist the surgeon in this matter, a computer assistance system has been developed facilitating navigation on the heart surface. After retrospective validation on patient data sets yielded good results, the system was successfully applied for in-vivo navigation on the front side of the heart during several CABG procedures. Postoperative evaluation confirmed that intraoperative navigation has been performed with adequate accuracy.
\end{abstract}

\section{Introduction}

The underlying pathology of CAD is intraluminal narrowing of the coronary arteries by wall plaque formations, severely limiting the blood flow to the myocardium. During CABG surgery, the blood flow is rerouted around a stenosis by a blood vessel graft. The position of the distal anastomosis on the diseased vessel is of great importance. Optimal placement of the anastomosis is a difficult task, especially as the target region may be covered with epicardial fat. To assist the heart surgeon in optimally placing the bypass graft, a computer assistance system facilitating navigation on the arrested heart during open heart CABG has been developed and tested.

\section{Materials and methods}

Nowadays, navigation systems are widely used in surgical disciplines such as neurosurgery and orthopaedic surgery [1,2]. Concerning cardiac interventions, valuable research has been done in minimally invasive surgery [3]. In open heart surgery, the development of navigation systems is still at the beginning. The Cardio-Pointer project is the first approach to develop a surgical assistance system for open heart CABG with navigation at the beating [4] and the arrested heart. The system concept comprises preoperative planning of the optimal position for the anastomosis as well as intraoperative navigation to that position. Navigation is facilitated by registration of pre- and intraoperative data of the patient's heart. This is illustrated in fig.1 which also shows the intraoperative setup and the surgeon using the Cardio-Pointer (trackable pointing device). 


\subsection{Preoperative data set and preoperative planning}

A cardiac MSCT (multi slice computed tomography) showing stenoses of the coronaries is recorded prior to surgery. For each diseased target vessel, the optimal distal anastomosis site is planned jointly by heart surgeon and diagnostic radiologist and marked in the MSCT data. Apart from forming the basis of the preoperative planning, the MSCT data are used as a basis for the registration and intraoperative visualisation. For the registration, 3D positions of coronary vessels and anatomical point landmarks potentially visible during surgery are extracted. For the visualisation, a volume rendered map of the coronaries is generated.

\subsection{Intraoperative data set and registration}

During surgery, the arrested heart is repositioned prior to grafting each bypass. As it is intended to represent the operating situs, intraoperative data have to be recorded after positioning the heart for subsequent bypass grafting. During the short period of data recording, registration and navigation, movement of heart or patient must be avoided. Intraoperative data are recorded with an infrared optical tracking system. Using the trackable Cardio-Pointer, the surgeon retraces visible parts of the vessels and points at anatomical point landmarks. In most patients, only a few point landmarks and vessel paths are actually visible on the heart surface due to epicardial fat. If the number of corresponding point landmarks is smaller than 3, additional landmarks can be generated in most cases. Then, all point landmarks are employed to coarsely match the data sets. The matching is then refined using the recorded vessel paths. Further improvement of the matching is achieved by a subsequent deformation correction. A more detailed description of the registration mechanism and the possibility of generating additional landmarks based on the vessel length was previously described in [5].
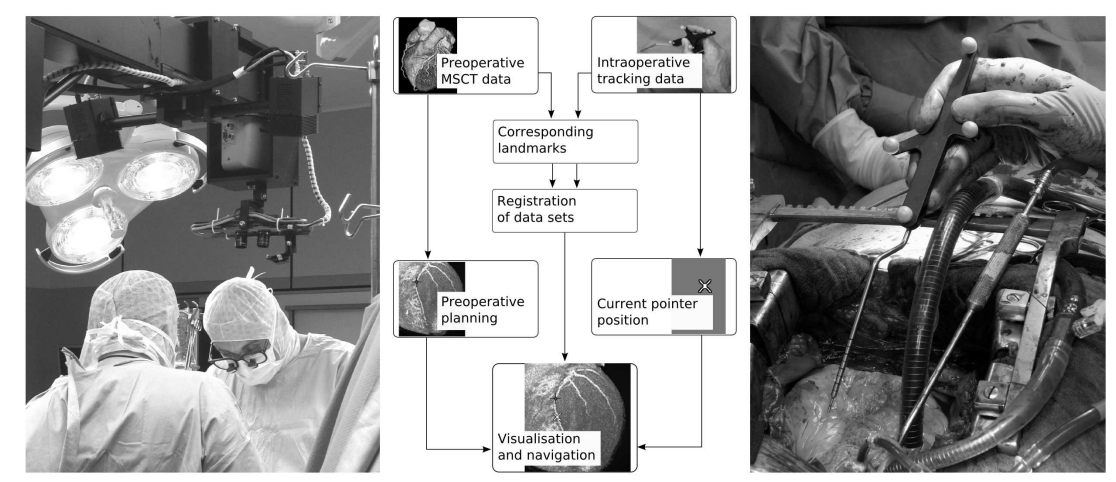

Fig. 1. From left: Intraoperative setup; Schematic view of the usage of pre- and intraoperative data for navigation; Cardio-Pointer on the heart surface. 
Table 1. Overall errors and standard deviation for 16 vessels and $48 \mathrm{LMs}$

\begin{tabular}{lr}
\hline Point landmarks & \\
\hline Mean RMS of point LMs (in $\mathrm{mm}$ ) & 2.4 \\
RMS standard deviation of point LMs (in $\mathrm{mm}$ ) & 1.0 \\
\hline Vessel paths & 1.6 \\
\hline Mean RMS of vessel paths (in $\mathrm{mm})$ & 1.0 \\
\hline
\end{tabular}

\subsection{Image-guided navigation}

Registration establishes a direct connection between the preoperative data including the planned anastomosis site and the intraoperative data representing the operating field. This allows for visualisation of the planned anastomosis site together with the current position of the pointer in the patient's coronary map. A video screen is used for visualisation. The displayed pointer position is continuously updated while the surgeon moves the pointer along the target vessel. Consequently, navigation to the preoperatively planned position is enabled.

\section{Results}

The registration mechanism developed for the arrested heart was validated in two phases: At first, intraoperative tracking data sets recorded during bypass grafting procedures have been registered retrospectively with preoperative CT data. The registration results obtained this way were very promising and justified subsequent in-vivo testing of the registration mechanism. Thus, the intraoperative modus operandi was altered to enable not only data recording but also immediate registration and subsequent navigation on the arrested heart.

\subsection{Evaluation of retrospective registration}

Retrospective registration was performed on 16 data sets from patients undergoing CABG surgery. Intraoperative data were recorded directly prior to bypass grafting after the heart was manually brought into a position exposing the target vessel in the centre of the operating field. Although considerable deformations of the heart were observed in each case, the registration mechanism for the arrested heart was able to match all data sets with sufficient accuracy, i.e. a similar accuracy is found for multiple measurements of the same anatomical landmark. 10 of the 16 data sets provided at least the minimally required number of 3 corresponding point landmarks, while 6 data sets didn't provide enough landmarks due to epicardial fat. For these data sets, 1 to 3 additional landmarks were generated on the basis of the unaltered vessel length. To evaluate the performance of the registration mechanism, both the RMS (root mean square) fiducial registration error of all corresponding landmark (LM) points and the average distance 
of corresponding vessel paths were calculated after registration (Tab. 1). Good results led to intraoperative live application of the system.

\subsection{Intraoperative registration and navigation}

Intraoperative navigation on target vessels on the front side of the heart has been performed during 7 bypass grafting procedures. Both, the complete registration mechanism and an alternative registration based on the vessel length [5] were used for live application. The resulting accuracies indicated no preference for either method. After successful registration, the surgeon performed navigation with the pointer on the arrested heart guided by the surgical assistance system. Target vessels on the front side of the heart were the LAD and its diagonal branches. In the patient's coronary map, the optimal position of the anastomosis as preoperatively planned by the heart surgeon and radiologist was displayed. After registration, the current position of the pointer was also displayed and continuously updated while the heart surgeon moved the pointer until its current position coincided with the planned anastomosis site (Fig. 2 (a)). This position was marked with a clip right beside the vessel and then, the bypass was grafted. Navigation on a target vessel including intraoperative data recording and registration takes about 3-4 minutes. During this short period of time, neither the heart nor the operating table must be moved. In two cases, successful registration was performed, but valid navigation was not possible due to unintendedly induced movement. This is attributed to the learning curve.To evaluate the navigation results, a postoperative MSCT of the patient was compared with the preoperative MSCT data. In both data sets, the same distinctive point on the target vessel path was used as reference point (Fig. 2b). The length of the vessel centreline from this reference point to the position of planned and grafted anastomosis respectively was determined. Comparison of both lengths shows how accurately the bypass was grafted to the preoperatively planned position. In case the position of the grafted anastomosis was not right beside the clip marking the navigation result, the clip position in the postoperative MSCT was evaluated. As a grafted anastomosis is several $\mathrm{mm}$ long, the centre of the anastomosis was
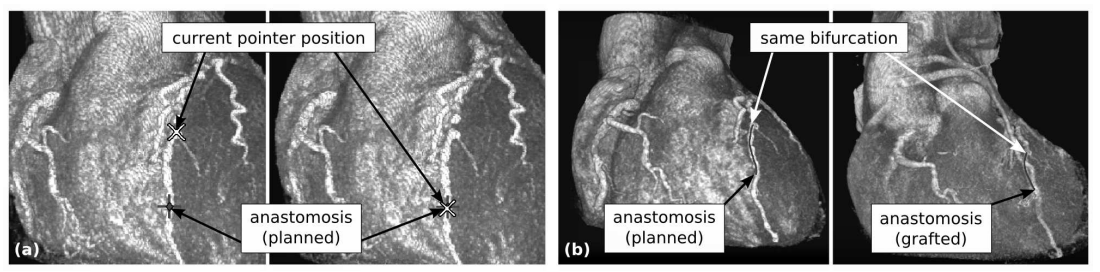

Fig. 2. (a) Intraoperative navigation: planned anastomosis site $($ dark +$)$ and current pointer position (bright $\mathrm{x}$ ) are displayed in the coronary map for one arbitrary moment during navigation and the end of navigation. (b) Evaluation of navigation result: preoperative and postoperative MSCTs with the distances from a bifurcation to the planned (left) and grafted (right) anastomosis respectively along the vessel centreline. 
Table 2. Navigation results

\begin{tabular}{llc}
\hline $\begin{array}{l}\text { Data } \\
\text { No. }\end{array}$ & $\begin{array}{l}\text { Target } \\
\text { vessel }\end{array}$ & $\begin{array}{c}\text { Distance from planned } \\
\text { to grafted site (in mm) }\end{array}$ \\
\hline 1 & LAD & 0.0 \\
2 & LAD & 3.1 \\
3 & LAD & 4.6 \\
4 & LAD & 2.2 \\
5 & LAD & 2.6 \\
6 & LAD & 3.4 \\
7 & D2 & 0.3 \\
\hline
\end{tabular}

Table 3. Target vessels and errors

\begin{tabular}{lr}
\hline Target vessels \\
\hline $\begin{array}{l}\text { Number of LAD bypass grafts } \\
\text { Number of bypass grafts on }\end{array}$ & 6 \\
diagonal branches of the LAD & 1 \\
\hline Navigation results & \\
\hline $\begin{array}{l}\text { Mean distance of planned and } \\
\text { grafted anastomosis (in mm) }\end{array}$ & 2.3 \\
$\begin{array}{l}\text { Standard deviation of the } \\
\text { distances (in mm) }\end{array}$ & 1.6 \\
\hline
\end{tabular}

used for calculations. Measurement inaccuracies of up to $\pm 2 \mathrm{~mm}$ may occur. For the 7 target vessels, the distance between planned and grafted anastomosis was $2.3 \pm 1.6 \mathrm{~mm}$, confirming good accordance of planned positions and navigation results (Tab. 2 and 3). This navigation accuracy actually reaches the limiting measurement accuracy at the arrested heart which amounts to $\pm 2.2 \mathrm{~mm}$.

\subsection{Discussion}

A surgical navigation system for computer assisted CABG on the arrested heart has been developed on patient data sets and its performance has been evaluated during several intraoperative live applications. The system successfully enabled intraoperative navigation on the surface of the heart during CABG surgery. The heart surgeon efficiently performed navigation with the Cardio-Pointer on the target vessels for bypass grafting. Postoperative evaluation shows that the intraoperative navigation correctly identified the planned anastomosis site in each case with sufficient accuracy. Navigation on all other target vessels for bypass grafting has been performed as well, the results currently being evaluated. The system developed within the Cardio-Pointer project is capable of matching pre- and intraoperative data sets from the patient's heart and provides precise intraoperative navigation assistance to a preoperatively planned position.

\section{References}

1. Peters TM. Image-guided surgery: From x-rays to virtual reality. Comput Meth Biomech Biomed Eng. 2000;4(1):27-57.

2. DiGioia AM, et al. Computer assisted navigation systems for hip and knee reconstructive surgery. In: DETEC. ASME 2001; 2001.

3. Coste-Manière E, et al. Optimal planning of robotically assisted heart surgery: First results on the transfer precision in the operating room. Int $\mathrm{J}$ Robot Res. 2004;23:539-548.

4. Hartung $\mathrm{C}$, et al. Image guidance for coronary artery bypass grafting. Int J CARS. 2008;3(6):505-510.

5. Gnahm C, et al. A registration approach for cardiac MSCT and optical tracking data recorded at the ischaemic heart. Proc MIUA; 2008. p. 251-255. 